\title{
Parameters Effect on Heat Transfer Augmentation in a Cavity with Moving Horizontal Walls
}

\author{
Doli Rani Pal1 ${ }^{*}$, Goutam Saha1, Kajal Chandra Saha² \\ ${ }^{1}$ Department of Mathematics, University of Dhaka, Dhaka, Bangladesh \\ ${ }^{2}$ Department of Applied Mathematics, University of Dhaka, Dhaka, Bangladesh \\ Email:*doli.math@du.ac.bd, gsahamath@du.ac.bd, kcsaha@du.ac.bd
}

How to cite this paper: Pal, D.R., Saha, G. and Saha, K.C. (2018) Parameters Effect on Heat Transfer Augmentation in a Cavity with Moving Horizontal Walls. Journal of Applied Mathematics and Physics, 6, 1907-1915.

https://doi.org/10.4236/jamp.2018.69162

Received: August 14, 2018

Accepted: September 22, 2018

Published: September 25, 2018

Copyright ( $\odot 2018$ by authors and Scientific Research Publishing Inc. This work is licensed under the Creative Commons Attribution International License (CC BY 4.0).

http://creativecommons.org/licenses/by/4.0/

\begin{abstract}
Mixed convection flow is one of the essential criteria of fluid flow and heat transfer. And its application has been increased due to modernization of society. So, to compete with the global world an analysis has been investigated numerically. In this study we have considered 2D double lid driven cavity with two-sided adiabatic walls. This problem is illustrated mathematically by a collection of governing equations and the developed model has been solved numerically by using Finite Difference Method (FDM). The goal of the present study is to analyze numerically the thermal behaviour and parameters effect on heat transfer inside the $2 \mathrm{D}$ chamber. Also this analysis has been observed for the case where the upper wall is moving at positive direction and lower wall is moving at negative direction with constant speed. Furthermore, we have tried to analyze the velocity and temperature profiles for a vast range of dimensionless parameters namely Reynolds number $(R e)$, Richardson number $(R i)$ and Prandtl number $(P r)$ and presented graphically. Moreover, it is found that these flow parameters have significant effects in controlling the flow behavior inside the cavity. A comparison has been done to validate our code and found a good agreement. Finally, average Nusselt number $(\mathrm{Nu})$ has been studied for the effects of these parameters and presented in tabular form.
\end{abstract}

\section{Keywords}

Mixed Convection, Nusselt Number, Lid-Driven Cavity, Heat Transfer, Shear Force 


\section{Introduction}

Mixed convection, takes place when natural convection and forced convection mechanisms perform simultaneously to exchange heat due to temperature differences. This is likewise characterized as circumstances where both pressure and buoyancy forces interface. The heat transfer behavior of the fluid as convection is mostly depended on the flow, temperature, geometry and inclination. In recent years, the natural and mixed convection flow has gained interest from their hypothetical and practical perspectives.

"The double lid-driven cavity problem has been gained extensive interest among the scientists and researchers because of its simplest geometrical settings with all fluid mechanical structure and applications such as cooling of electronic gadgets, drying instrument, softening procedures and so forth. Additionally, movement of side walls is also responsible for the fluid convective behavior within the enclosed square cavity" [1]. Furthermore, many numerical investigations are examined on double lid driven square cavities [2]-[15].

In recent past, $\mathrm{Pal}$ et al. [1] performed a numerical experiment on a lid driven cavity for low $R e$ flow using Finite Difference Method (FDM). Therefore, we observe that no investigation has been done on the behaviors of heat transfer filled with air/water inside a square enclosure where the upper and lower walls are moving at constant speed and side walls are adiabatic. In this study, we have considered low and high Reynolds number flow to examine heat transfer properties. In such manner, the goal of this investigation is to review the behavior of fluid and temperature fields for various $R e, R i$ and $P r$ in a double lid driven square domain. Moreover, average Nusselt number $(N u)$ is investigated in order to see the performance of the heat transfer inside the chamber.

\section{Governing Equation}

In the present experiment, a steady state, incompressible flow inside a cavity has been studied. The physical model along with boundary conditions is shown in Figure 1. To continue study, we have considered the case where the horizontal upper hot wall is moving towards and lower cold lid is moving backwards at a constant velocity. However, left and right side vertical wall are kept adiabatic in each case.

The following governing equation has been presented for 2D and incompressible flow in non-dimensional form:

$$
\begin{aligned}
& \frac{\partial U}{\partial X}+\frac{\partial V}{\partial Y}=0 \\
& U \frac{\partial U}{\partial X}+V \frac{\partial U}{\partial Y}=-\frac{\partial P}{\partial X}+\frac{1}{R e}\left(\frac{\partial^{2} U}{\partial X^{2}}+\frac{\partial^{2} U}{\partial Y^{2}}\right) \\
& U \frac{\partial V}{\partial X}+V \frac{\partial V}{\partial Y}=-\frac{\partial P}{\partial Y}+\frac{1}{R e}\left(\frac{\partial^{2} V}{\partial X^{2}}+\frac{\partial^{2} V}{\partial Y^{2}}\right)+R i \theta \\
& U \frac{\partial \theta}{\partial X}+V \frac{\partial \theta}{\partial Y}=\frac{1}{\operatorname{Pr} \operatorname{Re}}\left(\frac{\partial^{2} \theta}{\partial X^{2}}+\frac{\partial^{2} \theta}{\partial Y^{2}}\right)
\end{aligned}
$$




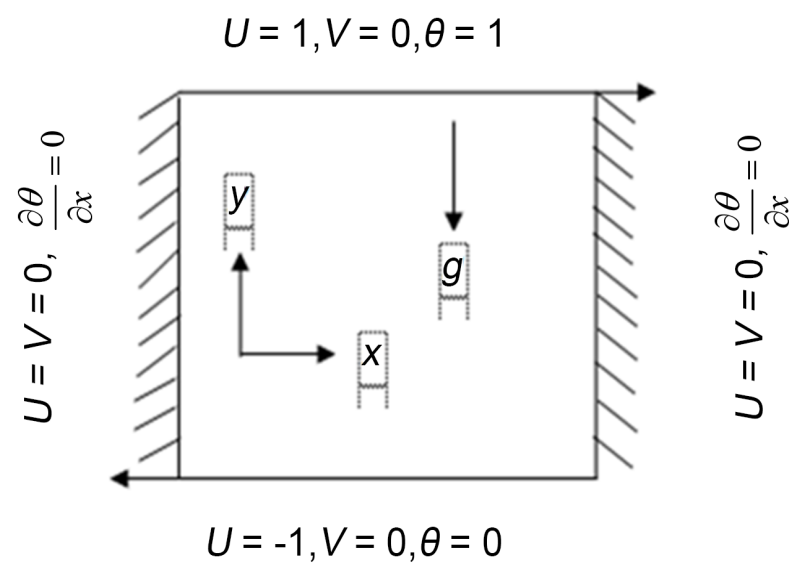

Figure 1. Simplified diagram of the physical geometry.

where $U$ and $V$ are the velocity components along $X$ and $Y$ directions, $\theta$ is the non-dimensional temperature and $P$ is the non-dimensional pressure component. Here, the dimensionless parameters in Equations (1)-(4) are: $R i$ is the Richardson number, $R e$ is the Reynolds number, $G r$ is the Grashof number, $P r$ is the Prandtl number. The governing parameters in Equations (1)-(4) and non-dimensional length and velocities are as follow:

$$
\begin{gathered}
X=\frac{x}{L} ; Y=\frac{y}{L} ; U=\frac{u}{V_{L}} ; V=\frac{v}{V_{L}} ; P=\frac{p}{\rho V_{L}^{2}} ; \theta=\frac{T-T_{C}}{T_{H}-T_{C}} ; \\
R i=\frac{G r}{R e^{2}} ; \operatorname{Re}=\frac{V_{L} L}{v} ; G r=\frac{g \beta \Delta T L^{3}}{v^{3}} ; \operatorname{Pr}=\frac{v}{\alpha} ;
\end{gathered}
$$

where $\rho, g, \alpha, \beta$ and $v$ are the fluid density, gravitational acceleration, thermal diffusivity, the coefficient of thermal expansion and kinematic viscosity, respectively. The velocity and temperature flow fields boundary conditions are shown in Figure 1 and have the following dimensionless form:

$$
\left.\begin{array}{ll}
U=V=0, \frac{\partial \theta}{\partial x}=0 & \text { at } X=0 \text { and } X=1 \\
U=-1, V=0, \theta=0 & \text { at } Y=0 \\
U=1, V=0, \theta=1 & \text { at } Y=1
\end{array}\right\}
$$

Also, the definition of local and average Nusselt number is described in Pal et al. [1].

\section{Numerical Procedure and Validation}

We have used finite difference method to deal with the governing equations (1)-(4) along with the boundary situation (5). Then an in house code (DGK) has been implemented to represent the flow and thermal behaviors. However, grid independent test has been done to verify the code which is also presented in Pal et al. [1]. Furthermore, numerical approximation and comparison has been examined graphically for centerlines velocity profiles with Adair et al. [16], Ghia et al. [9] and found good harmony. Another study has been presented for average 
Nusselt number by using Simpson's 1/3 rule to approve the code and validate result with Abu-Nada et al. [7], Waheed et al. [5] and Sharif et al. [17]. Aspects of these studies have been discussed in Pal et al. [1].

\section{Result and Discussion}

In this research, variation of velocity and temperature profiles with different $R e$, $R i$ and $P r$ inside the square cavity with the given boundary conditions (5) are studied and explained graphically in Figures 2-4. Additionally, variation of average Nusselt number has been studied and presented in tabular form in Table 1. The dimensionless parameters considered here are $100 \leq R e \leq 1000,0.1 \leq R i \leq$ 3.0 and $0.71 \leq \operatorname{Pr} \leq 10$.

The flow and thermal fields within the double lid driven cavity for $R i=1.5$ and $\operatorname{Pr}=10.0$ are represented in Figure 2 for numerous values of Reynolds number. This profiles shows that for $R e=500$ fluid flow develops a high speed velocity region near the moving lid. For the moving lids, there appear two large secondary vortices in the two opposite corner and two tiny secondary vortices in the center of the cavity. The flow is dominated by the forced convection and due to high temperature difference along the moving lids, the flow field becomes more turbulent. Furthermore, the size of the secondary vortices increases with the increase of $R e$ to 1000 . Moreover, two tiny secondary eddies as it is located at the center of the cavity form another convective cell inside it which seems to be chaotic. From temperature profiles in Figure 2, there occurs high temperature

Table 1. Variation of average Nusselt number for different Re, Pr and Ri.

\begin{tabular}{|c|c|c|c|}
\hline$R e=100$ & & Avg Nu & \\
\hline$P r$ & $R i=0.1$ & $R i=1.0$ & $R i=1.5$ \\
\hline 0.71 & 4.15 & 1.84 & 1.52 \\
\hline 2 & 7.58 & 2.69 & 2.09 \\
\hline 5 & 11.89 & 4.00 & 2.86 \\
\hline 10 & 15.62 & 6.09 & 3.77 \\
\hline \multicolumn{4}{|l|}{$R e=500$} \\
\hline$P r$ & $R i=0.1$ & $R i=1.0$ & $R i=1.5$ \\
\hline 0.71 & 9.24 & 2.04 & 1.81 \\
\hline 2 & 15.47 & 3.12 & 2.63 \\
\hline 5 & 21.69 & 4.85 & 3.93 \\
\hline 10 & 33.68 & 7.41 & 5.20 \\
\hline \multicolumn{4}{|l|}{$R e=1000$} \\
\hline$P r$ & $R i=0.1$ & $R i=1.0$ & $R i=1.5$ \\
\hline 0.71 & 11.98 & 2.12 & 1.82 \\
\hline 2 & 16.95 & 3.29 & 2.67 \\
\hline 5 & 20.59 & 5.05 & 4.21 \\
\hline 10 & 31.25 & 8.11 & 5.64 \\
\hline
\end{tabular}




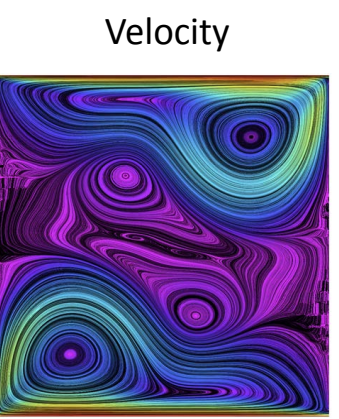

Temperature

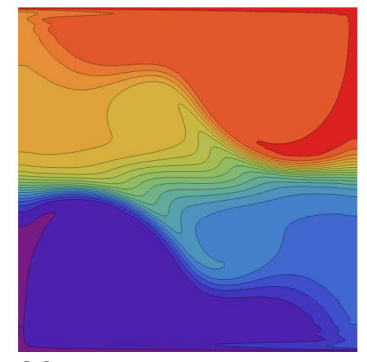

$\mathrm{Re}=500$

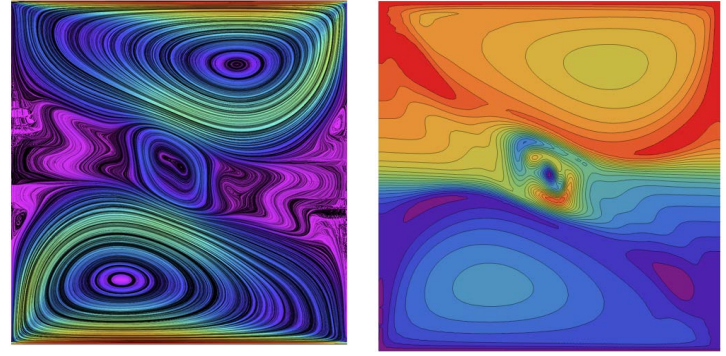

$\operatorname{Re}=1000$

Figure 2. Variation of velocity and temperature profiles with different Reynolds number for $R i=1.5, \operatorname{Pr}=10.0$.
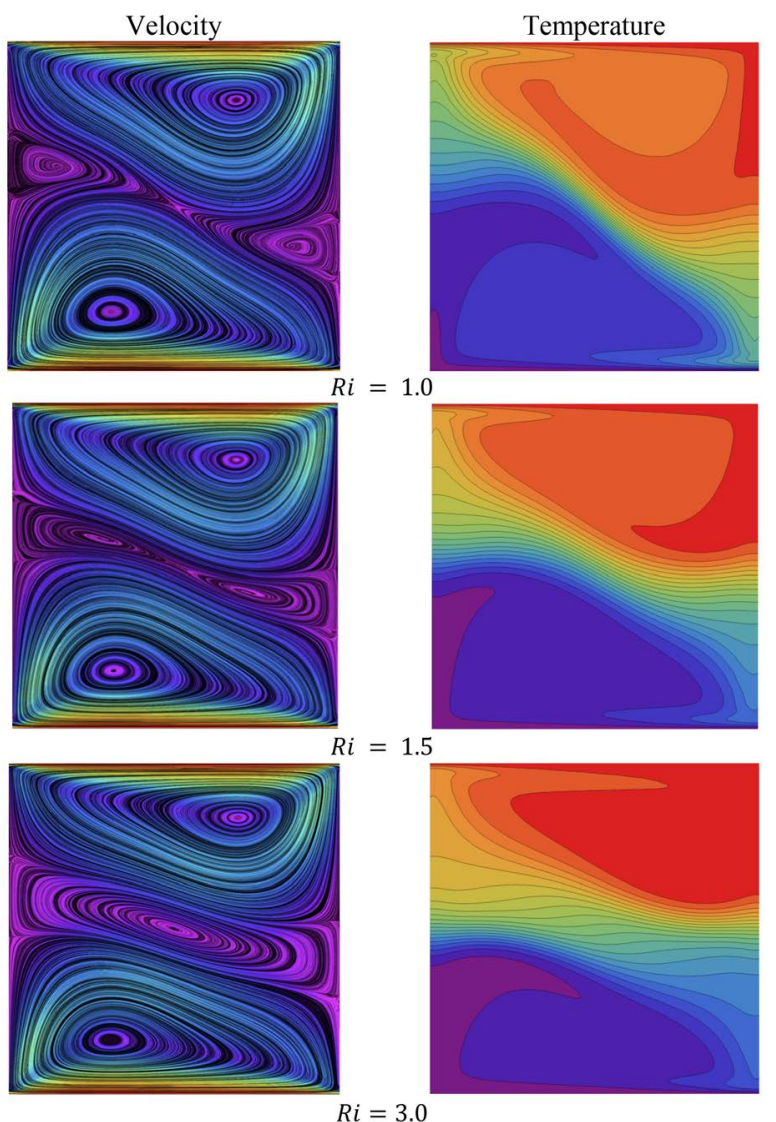

$$
R i=3.0
$$

Figure 3. Variation of velocity and temperature profiles with different Richardson number for $R e=100, P r=10.0$. 


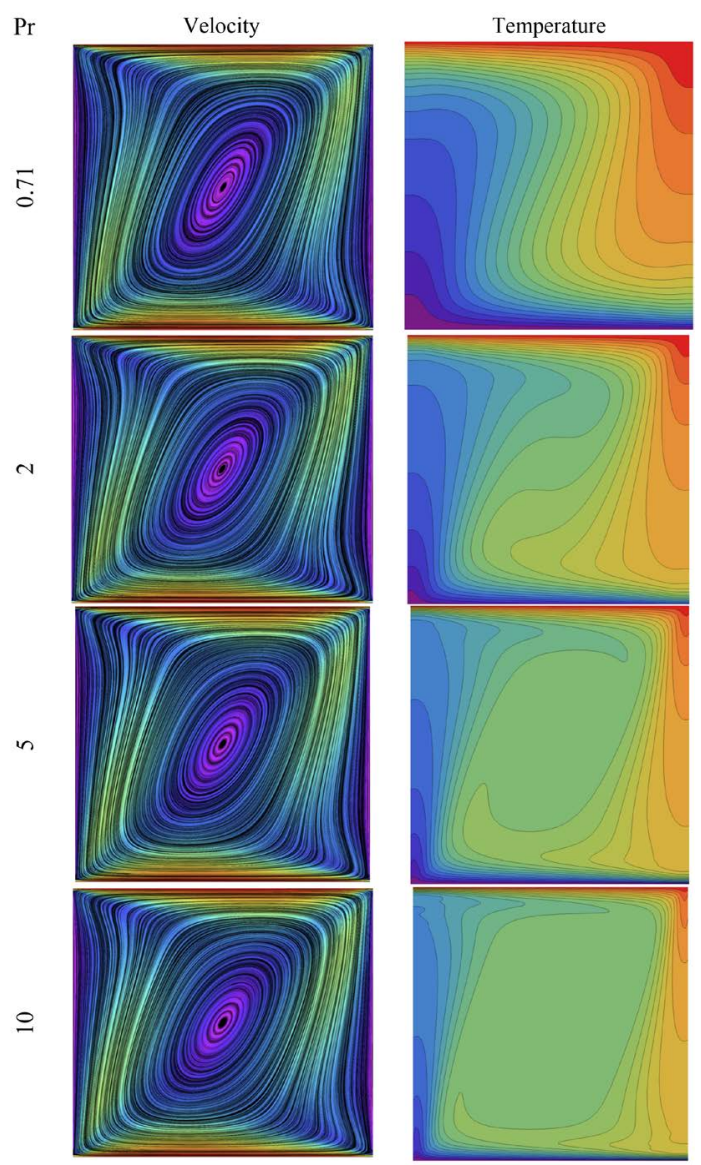

Figure 4. Variation of velocity and temperature with different Prandtl number for $R e=100, R i=0.1$.

difference region near the moving lid for $R e=500$. Additionally, the center thermal region separate into two parts. One part moving towards the hot region and another part moving towards the cold region. These all makes a steep temperature gradient along the adiabatic wall. This is due to the presence of shear stress. As an increase of Reynolds number $R e=1000$, shows hot fluid mixes up with the cold fluid very well and the thermal boundary layer diminishes.

Figure 3 represents the variation of velocity and isothermal profiles for miscellaneous values of $R i$ with fixed $R e=100$ and $P r=10.0$. Usually, the flow seems to be dominated by the mixed convection for $0.1<R i<10$. From velocity profiles for $R i=1.0$, it is observed that the upper and lower secondary cells are moving in the clockwise pattern due to the movement of the upper and lower walls. Moreover, it creates high speed velocity region near the velocity boundary layer and it covers most of the cavity. However, two small eddies have been visible near the vertical walls. Besides, the tiny secondary vortices tend to merge and finally form a large secondary vortex for $R i=1.5,3.0$ in the core of the cavity which may control the flow field with further increment of $R i$. As a result, dominant secondary vortices are decreased in size. In this regard, buoyancy effect is clearly seen in velocity profiles. From Figure 3 , it is seen that shear powers are 
prevailing in the cavity and, subsequently, the isotherms are generally assemble to the region close to the top and bottom surfaces of the square cavity for $R i=$ 1.0. The high temperature inclination close to the upper and lower walls is because of these high shear stress created due to moving lids. Also, a thin thermal boundary layer has developed on the top of the hot lid. Besides, mid temperature zone is visible in the center of the domain. Now if we look forward, expanding $R i$ up to 1.5 , the shear forces and buoyancy forces are a similar extent. In this way, coarse isotherms existed close to the top and base walls. Therefore, a direct temperature difference in the vertical direction can be seen. Additionally, increments in $R i(R i=3.0)$ prompt more extensive development of isotherms covering the entire cavity. This leads a further abatement of the heat exchange strength the along moving lid because of a dynamically stable free convection impact. Along these lines, the buoyancy force characterizes the shaped fluid stream and heat exchange inside the domain.

Figure 4 represents the isothermal profiles for various $\operatorname{Pr}$ such as $\operatorname{Pr}=0.71$, 2.0, 5.0, 10.0 and taking the other dimensionless numbers such as $R e=100$ and $R i=0.10$ as constant. If we consider the velocity profiles in Figure 4 for various Prandtl number then it is observed that velocity profiles is independent of Prandtl number. There exists a primary eddy for $P r=0.71$ and when $P r$ increases for the next three cases, there is no significant differences on the flow field. Because for $R i<1.0$ the flow is commanded by the shear force inside cavity and determines the temperature and buoyancy variations in the cavity. It shows strong dependency on Pr. From Figure 4, it is seen that there is a development of higher temperature difference zones located near the two opposite corners due to the adiabatic walls. As we increase the Prandtl number ( $P r$ $=2.0$ ), the temperature patterns are trying to twist due to the forced convection. Moreover, the thermal boundary layer thickness decreases so the average $N u$ increases with the higher values of Pr. In addition there exists a large isotherm region in the core of the cavity and tend to increase if $P r$ increases more.

Table 1 represent the average $N u$ for wide range of different dimensionless parameters such as $R e, \operatorname{Pr}$ and $R i$. Further, it is seen that percentage enhancements of avg. $\mathrm{Nu}$ are $45 \%, 65 \%$ and $73 \%$ respectively for $R e=100$ and $\operatorname{Pr}=2,5$, 10. Moreover, such percentage enhancements of avg. $\mathrm{Nu}$ are $74 \%, 80 \% 88 \%$ respectively for $R e=500$ and $P r=2,5,10$. Also increment in percentage of the rate of heat transfer are $76 \% 80 \% 87 \%$ where $R e=1000$ and $P r=2,5,10$, in all these cases keeping $R i=0.1$. More precisely, the maximum heat transfer difference almost $10 \%$ occurs when we consider $R e=100$ and $R e=500$. After that, it reduces to almost $2 \%$ to $3 \%$ between $R e=500$ to $R e=1000$. Thus we can conclude that if $\mathrm{Pr}$ increase then the heat transfer rate increases. Also, highest heat transfer rate occurs for small values of $R i$. Highest heat exchange attribute occurs for $R i=0.1$ which is fallen by almost $40 \%$ for $R i=1.0$. Finally, the heat transfer rate slightly decreases with the increase of $R i$. 


\section{Conclusion}

The flow phenomena and heat transfer are observed inside a bounded domain where the upper wall is moving in the positive direction and lower wall is moving in the negative direction. The geometrical characteristics have been set to the Equations (1)-(5) and then solved by 4th order FDM. The effects of dimensionless parameters $R e$, and Pr have been presented graphically and in tabular form. It is noticed that there is no effect of $\operatorname{Pr}$ on the velocity profiles but strong effect on temperature profiles. Also, it is found that for increasing of $R i$, rate of heat exchange decreases. Besides, for low Richardson number flow is dynamically unstable and for higher values of $R i$, the flow is stable. Furthermore, as $R e$ increases, the augmentation of average $N u$ increases for all cases. Overall, higher intensification of average $\mathrm{Nu}$ is pragmatic when flow becomes turbulent and deterioration of average $\mathrm{Nu}$ can be realistic when flow becomes laminar.

\section{Acknowledgements}

This work has been fully supported by University Grant Commission (UGC), Bangladesh via grant number Reg./Admin-3/76338 (Year: 2017-2018).

\section{Conflicts of Interest}

The authors declare no conflicts of interest regarding the publication of this paper.

\section{References}

[1] Pal, D.R., Saha, G. and Saha, K.C. (2018) A Case Study of Double Lid Driven Cavity for Low Reynolds Number Flow. Dhaka University Journal of Science, 66, 95-101.

[2] Ramanan, N. and Homsy, G.M. (1994) Linear Stability of Lid-Driven Cavity Flows. Physics of Fluids, 6, 2690-2701. https://doi.org/10.1063/1.868158

[3] Iwatsu, R., Hyun, J.M. and Kuwahara, K. (1992) Convection in a Differentially Heated Square Cavity with a Torsionally-Oscillating Lid. International Journal of Heat and Mass Transfer, 35, 1069-1076. https://doi.org/10.1016/0017-9310(92)90167-Q

[4] Iwatsu, R., Hyun, J.M. and Kuwahara, K. (1992) Numerical Simulation of Flows Driven by a Torsionally-Oscillating Lid in a Square Cavity. Journal of Fluids Engineering, 114, 143-149. https://doi.org/10.1115/1.2910008

[5] Waheed, M.A. (2009) Mixed Convective Heat Transfer in Rectangular Enclosures Driven by a Continuously Moving Horizontal Plate. International Journal of Heat and Mass Transfer, 52, 5055-5063. https://doi.org/10.1016/j.ijheatmasstransfer.2009.05.011

[6] Chen, C.J., Nassari-Neshat, H. and Ho, K.S. (1981) Finite-Analytical Numerical Solution of Heat Transfer in Two Dimensional Cavity Flow. Numerical Heat Transfer, 4, 179-197. https://doi.org/10.1080/01495728108961786

[7] Abu-Nada, E. and Chamkha, A.J. (2010) Mixed Convection Flow in a Lid-Driven Inclined Square Enclosure Filled with a Nanofluid. European Journal of Mechanics-B/Fluids, 29, 472-482. https://doi.org/10.1016/j.euromechflu.2010.06.008

[8] Aydin, O. (1999) Aiding and Opposing Mechanisms of Mixed Convection in a 
Shear and Buoyancy Driven Cavity. International Communications in Heat and Mass Transfer, 26, 1019-1028. https://doi.org/10.1016/S0735-1933(99)00091-3

[9] Ghia, U., Ghia, K.N. and Shin, C.T. (1982) High-Resolutions for Incompressible Flow Using the Navier-Stokes Equations and a Multi-Grid Method. Journal of Computational Physics, 48, 387-411. https://doi.org/10.1016/0021-9991(82)90058-4

[10] Moallemi, M.K. and Jang, K.S. (1992) Prandtl Number Effects on Laminar Mixed Convection Heat Transfer in a Lid-Driven Cavity. International Journal of Heat and Mass Transfer, 35, 1881-1892. https://doi.org/10.1016/0017-9310(92)90191-T

[11] Hakan, F. and Oztop, I.D. (2004) Mixed Convection in Two-Sided Lid Driven Differentially Heated Square Cavity. International Journal of Heat and Mass Transfer, 47, 1761-1769. https://doi.org/10.1016/j.ijheatmasstransfer.2003.10.016

[12] Chamkha, A.J. (2002) Hydro-Magnetic Combined Convection Flow in a Vertical Lid-Driven Cavity with Internal Heat Generation or Absorption. Numerical Heat Transfer, Part A: Applications, 41, 529-546. https://doi.org/10.1080/104077802753570356

[13] Hussain, S. (2017) Effects of Inclination Angle on Mixed Convective Nanofluid Flow in a Double Lid-Driven Cavity with Discrete Heat Sources. International Journal of Heat and Mass Transfer, 106, 847-860. https://doi.org/10.1016/j.ijheatmasstransfer.2016.10.016

[14] Sivasankaran, V.S. (2013) Numerical Study on Mixed Convection in an Inclined Lid-Driven Cavity with Discrete Heating. International Communications in Heat and Mass Transfer, 46, 112-125.

https://doi.org/10.1016/j.icheatmasstransfer.2013.05.022

[15] Malik, A.N. (2016) A Comparative Study of Mixed Convection and Its Effects on Partially Active Thermal Zones in a Two Sided Lid-Driven Cavity Filled with Nanofluid. Engineering Science and Technology, an International Journal, 19, 1283-1298. https://doi.org/10.1016/j.jestch.2016.02.008

[16] Adair, M.J. (2015) Developing an Understanding of the Steps Involved in Solving Navier-Stokes Equations. The Mathematica Journal, 17, 1-19. https://doi.org/10.3888/tmj.17-8

[17] Sharif, M.A.R. (2007) Laminar Mixed Convection in Shallow Inclined Driven Cavities with Hot Moving Lid on Top and Cooled from Bottom. Applied Thermal Engineering, 27, 1036-1042. https://doi.org/10.1016/j.applthermaleng.2006.07.035 